\title{
Application of the modified exponential function method to Vakhnenko-Parkes equation
}

\author{
Gülnur Yele,*, Tolga Aktürk ${ }^{b}$ \\ ${ }^{a}$ Faculty of Educational Sciences, Final International University, Kyrenia, Mersin 10, Turkey. \\ ${ }^{b}$ Department of Mathematics and Science Education, Faculty of Education, Ordu University, Turkey.
}

\begin{abstract}
In this paper, we submit some new travelling wave solutions for the Vakhnenko-Parkes equation via the modified exponential function method. The obtained solutions include hyperbolic, exponential, trigonometric function solutions. Regarding these solutions, the 2D and 3D graphs and contour simulations are presented.
\end{abstract}

Keywords: The nonlinear evolution equations, the Vakhnenko-Parkes equation, the modified exponential function method. 2010 MSC: 35C07, 35D99, 65K99.

(C)2020 All rights reserved.

\section{Introduction}

Nonlinear partial differential equations have an important place in other sciences such as physics and engineering. There are various methods in the literature to find solutions to such equations. Some of these are as follows; the trial equation method [35, 38], the extended trial equation method [47, 48], the new function method [1, 2, 14], the generalized Bernoulli sub-equation function method [8, 16, 54], Kudryashov's method [33, 39], the sine-Gordon expansion method [10, 21, 34, 55], Hirota bilinear method $[28,50,51]$ and so on. In this study, we apply the modified exponential function method (MEFM) to the Vakhnenko-Parkes (VP) equation. Vakhnenko and Parkes have obtained an exact implicit N-soliton solution by Hirota method. To construct conservation laws, a Backlund transformation is used. The standart inverse scattering transform method has gave M-mode periodic solutions. In the other study, a variable separation solution with two arbitrary functions is obtained and the soliton-type, instanton-type and rogue wave-type structures are presented [61]. The many scientist's studies on the Vakhnenko-Parkes (VP) equation via various methods can be seen at [29, 36, 42, 44, 49].

$$
u u_{x x t}-u_{x} u_{x t}+u^{2} u_{t}=0 .
$$

The Vakhnenko equation (VE) models the propagation of high-frequency waves in a relaxing medium. The Vakhnenko-Parkes equation is a non-linear equation which is an alternative form of VE.

\footnotetext{
${ }^{*}$ Corresponding author

Email addresses: gulnur.yel@final.edu.tr (Gülnur Yel), tolgaakturkk@gmail.com (Tolga Aktürk)

doi: $10.22436 / \mathrm{mns} .06 .01 .02$
}

Received: 2019-08-01 Revised: 2019-09-27 Accepted: 2019-12-06 
This paper is organized as follows: In the 2nd Section, we present the general structure of the modified exponential function method. In Section 3, the application of the purposed method to Vakhnenko-Parkes equation and all figures obtained from solutions are presented. In the last section, we present some conclusions.

\section{Analysis of the Method}

MEFM has the three steps that can be summarized as follows [9, 13, 30, 53].

Let consider the nonlinear partial differential equations to apply this method as follows;

$$
P\left(u, u_{x}, u_{t}, u_{x x}, u_{t t}, u_{t x}, \cdots\right)=0,
$$

where $u=u(x, t)$ is unknown function, $P$ is a polynomial that has $u(x, t)$ function and its partial derivatives respect to $x$ and $t$.

Step 1: Suppose that the traveling wave transformation as,

$$
\mathrm{u}(x, \mathrm{t})=\mathrm{u}(\zeta), \zeta=\mathrm{k}(x-\mathrm{ct}),
$$

where $k, c$ are a nonzero constants that can be determined later. Using partial derivatives of the Eq. (2.2) into Eq. (2.1), the Eq. (2.1) is converted to a nonlinear ordinary differential equation defined as;

$$
\mathrm{N}\left(\mathrm{U}, \mathrm{U}^{\prime}, \mathrm{U}^{\prime \prime}, \mathrm{u}^{\prime \prime \prime}, \cdots\right)=0,
$$

where $\mathrm{N}$ is a polynomial depend on $\mathrm{U}$.

Step 2: We suppose the traveling wave solution of Eq. (2.3) can be expressed as follows;

$$
u(\zeta)=\frac{\sum_{i=0}^{N} A_{i}[\exp (-\Omega(\zeta))]^{i}}{\sum_{j=0}^{M} B_{j}[\exp (-\Omega(\zeta))]^{j}}=\frac{A_{0}+A_{1} \exp (-\Omega)+\cdots+A_{N} \exp (N(-\Omega))}{B_{0}+B_{1} \exp (-\Omega)+\cdots+B_{M} \exp (M(-\Omega))^{\prime}},
$$

where $A_{i}, B_{j},(0 \leqslant i \leqslant N, 0 \leqslant j \leqslant M)$ are constants can be determined later, $A_{N} \neq 0, B_{M} \neq 0$ and $\Omega=$ $\Omega(\zeta)$ provides the following ordinary differential equation;

$$
\Omega^{\prime}(\zeta)=\exp (-\Omega(\zeta))+\mu \exp (\Omega(\zeta))+\lambda
$$

When we solve the Eq. (2.5), we reach the five solution families as follows [7, 15]:

Family 1: When $\mu \neq 0, \lambda^{2}-4 \mu>0$,

$$
\Omega(\zeta)=\ln \left(\frac{-\sqrt{\lambda^{2}-4 \mu}}{2 \mu} \tanh \left(\frac{\sqrt{\lambda^{2}-4 \mu}}{2}(\zeta+E)\right)-\frac{\lambda}{2 \mu}\right) .
$$

Family 2: When $\mu \neq 0, \lambda^{2}-4 \mu<0$,

$$
\Omega(\zeta)=\ln \left(\frac{\sqrt{-\lambda^{2}+4 \mu}}{2 \mu} \tan \left(\frac{\sqrt{-\lambda^{2}+4 \mu}}{2}(\zeta+E)\right)-\frac{\lambda}{2 \mu}\right) .
$$

Family 3: When $\mu=0, \lambda \neq 0$ and $\lambda^{2}-4 \mu>0$,

$$
\Omega(\zeta)=-\ln \left(\frac{\lambda}{\exp (\lambda(\zeta+E))-1}\right) .
$$

Family 4: When $\mu \neq 0, \lambda \neq 0$ and $\lambda^{2}-4 \mu=0$,

$$
\Omega(\zeta)=\ln \left(-\frac{2 \lambda(\zeta+E)+4}{\lambda^{2}(\zeta+E)}\right) .
$$

Family 5: When $\mu=0, \lambda=0$ and $\lambda^{2}-4 \mu=0$,

$$
\Omega(\zeta)=\ln (\zeta+E) .
$$

where $A_{0}, A_{1}, \ldots, A_{N}, B_{0}, B_{1}, \ldots, B_{M}, E, \lambda, \mu$ are constants and can be determined later. Using homogenous balance principle between the highest nonlinear terms with the highest orderderivatives of $U$ in Eq. (2.4) it can be find a relationship between $\mathrm{N}$ and $\mathrm{M}$. 
Step 3: Substituting Eq. (2.5) along with solution families into Eq. (2.4) we have a polynomial of $\exp (\Omega(\xi))$. After all coefficients of the similar power of $\exp (\Omega(\xi))$ are equated to zero, it yields an algebraic equation system in terms of $A_{0}, A_{1}, \ldots, A_{N}, B_{0}, B_{1}, \ldots, B_{M}, E, \lambda, \mu$. At the end of this procedure, the obtained values of coefficients substituting into Eq. (2.4), it provides the traveling wave solutions of Eq. (2.1).

\section{Application}

Let use Eq. (2.2) along with Eq. (2.4) into Eq. (1.1). In this case, we have nonlinear ordinary differential equation as follows;

$$
k^{2} u u^{\prime \prime}-c k^{2}\left(u^{\prime}\right)^{2}+\frac{1}{3} u^{3}=0
$$

When applying the balance principle (3.1), which is the highest-order derivative containing the term $\mathrm{UU}^{\prime \prime}$ and the non-linear $\mathrm{U}^{3}$ term, the following (3.2) relation is obtained,

$$
\mathrm{M}+2=\mathrm{N} .
$$

For suitable integer values of $M$ and $N$, one can achieve different cases. We have chosen $M=1$ and $N=3$ values to Eq. (2.4), $\mathrm{U}, \mathrm{U}^{\prime}, \mathrm{U}^{\prime \prime}$ can be written as,

$$
\begin{aligned}
& \mathrm{U}(\zeta)=\frac{\psi}{\varphi}=\frac{\mathrm{A}_{0}+\mathrm{A}_{1} \mathrm{e}^{-\Omega(\zeta)}+\mathrm{A}_{2} \mathrm{e}^{-2 \Omega(\zeta)}+\mathrm{A}_{3} \mathrm{e}^{-3 \Omega(\zeta)}}{\mathrm{B}_{0}+\mathrm{B}_{1} \mathrm{e}^{-\Omega(\zeta)}}, \\
& \mathrm{U}^{\prime}(\zeta)=\frac{\psi^{\prime} \varphi-\psi \varphi^{\prime}}{\varphi^{2}}, \\
& \mathrm{U}^{\prime \prime}(\zeta)=\frac{\psi^{\prime \prime} \varphi^{3}-\varphi^{2} \psi^{\prime} \varphi^{\prime}-\left(\psi \varphi^{\prime \prime}+\psi^{\prime} \varphi^{\prime}\right) \varphi^{2}+2\left(\psi^{\prime}\right)^{2} \psi \varphi}{\varphi^{4}} .
\end{aligned}
$$

If we consider Eq. (3.3) into Eq. (3.1), some travelling wave solutions have arisen as following submitted.

\section{Case 1:}

$$
A_{0}=-6 k^{2} \mu B_{0}, A_{1}=\mu A_{3}-6 k^{2} \lambda B_{0}, A_{2}=\lambda A_{3}-6 k^{2} B_{0}, B_{1}=-\frac{A_{3}}{6 k^{2}} .
$$

Using the above coefficients, we have the following two solution families.

\section{Family 1:}

$$
u_{1,1}(x, t)=\frac{6 k^{2}\left(\lambda^{2}-4 \mu\right) \mu}{\left(\lambda \operatorname{Cosh}\left[\frac{1}{2}(E+k(-c t+x)) \sqrt{\lambda^{2}-4 \mu}\right]+\sqrt{\lambda^{2}-4 \mu} \operatorname{Sinh}\left[\frac{1}{2}(E+k(-c t+x)) \sqrt{\lambda^{2}-4 \mu}\right]\right)^{2}} .
$$
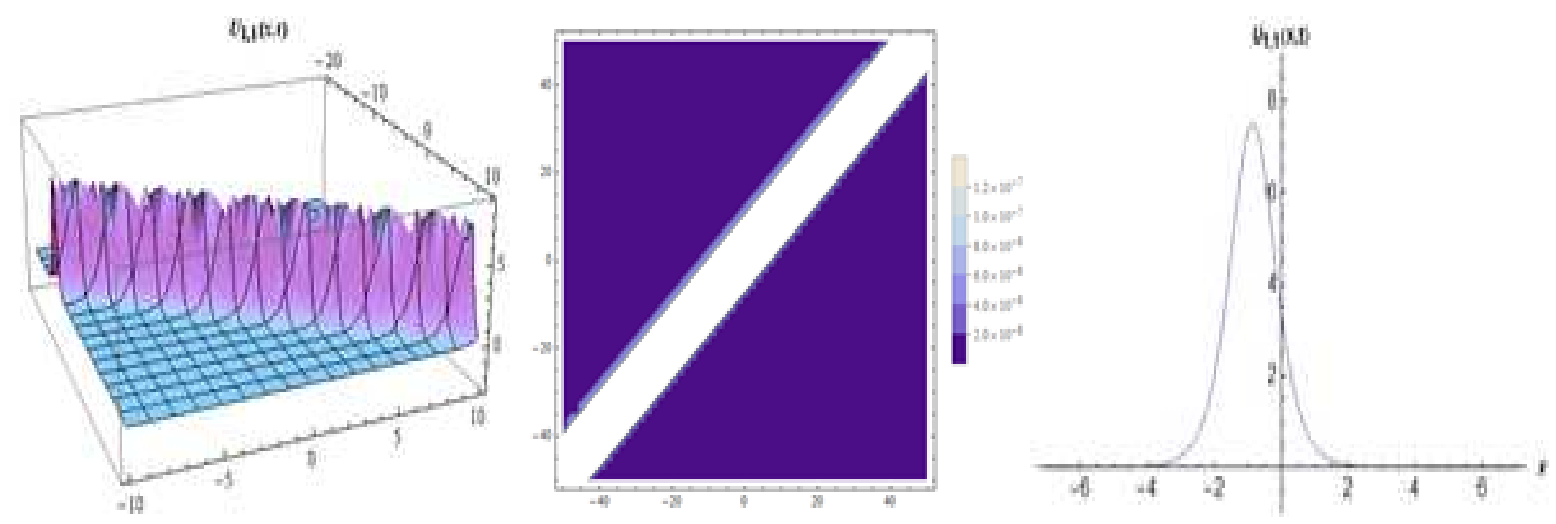

Figure 1: The 3D and contour graphs and $t=1$ for $2 \mathrm{D}$ of Eq. (3.5).

\section{Family 2:}

$$
u_{1,2}(x, t)=\frac{6 k^{2}\left(\lambda^{2}-4 \mu\right) \mu}{\left(\lambda \operatorname{Cos}\left[\frac{1}{2}(E+k(-c t+x)) \sqrt{-\lambda^{2}+4 \mu}\right]-\sqrt{-\lambda^{2}+4 \mu} \operatorname{Sin}\left[\frac{1}{2}(E+k(-c t+x)) \sqrt{-\lambda^{2}+4 \mu}\right]\right)^{2}} .
$$



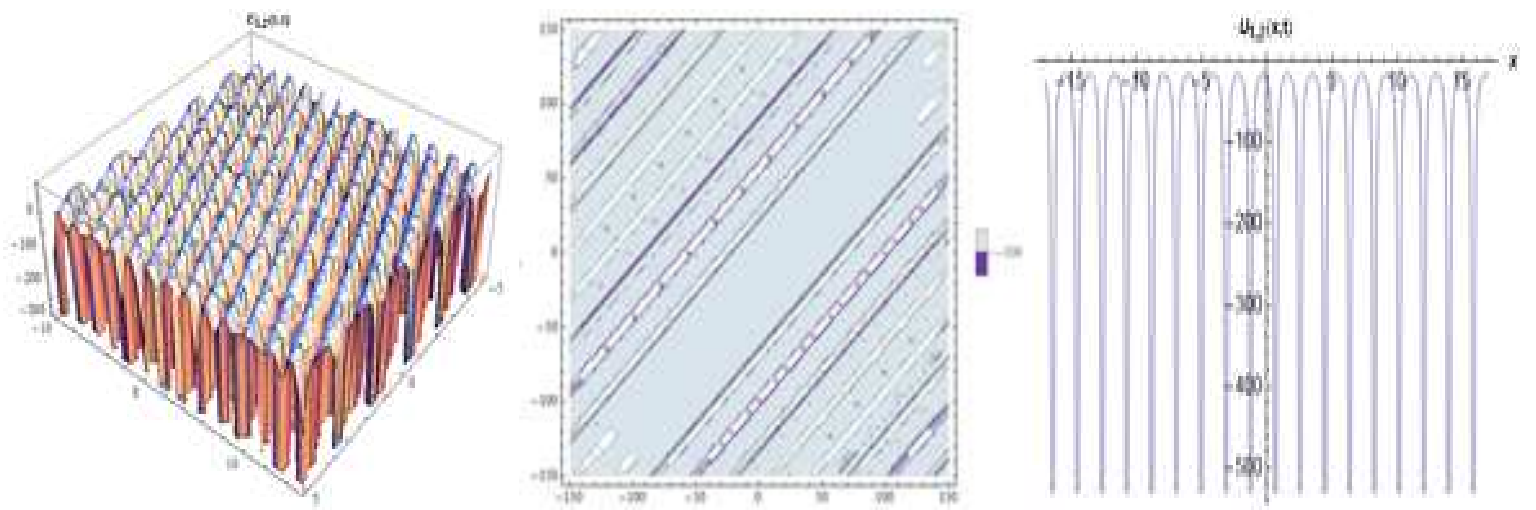

Figure 2: The $3 \mathrm{D}$ and contour graphs and $t=1$ for $2 \mathrm{D}$ of Eq. (3.6).

\section{Case 2:}

\section{Family 2:}

$$
A_{0}=\frac{\mu A_{3} B_{0}}{B_{1}}, A_{1}=A_{3}\left(\mu+\frac{\lambda B_{0}}{B_{1}}\right), A_{2}=A_{3}\left(\lambda+\frac{B_{0}}{B_{1}}\right), k=i \sqrt{\frac{A_{3}}{6 B_{1}}} .
$$

The $A_{0}, A_{1}, A_{2}, A_{3}, k$ coefficients enable to write the following two solution families.

\section{Family 1:}

$$
u_{2,1}(x, t)=\frac{\mu A_{3}\left(-\lambda^{2}+4 \mu\right)}{\left(\lambda \operatorname{Cosh}[f(x, t)]+\sqrt{\lambda^{2}-4 \mu} \operatorname{Sinh}[f(x, t)]\right)^{2} B_{1}},
$$

where $f(x, t)=\frac{1}{2} \sqrt{\lambda^{2}-4 \mu}\left(E+\frac{i(-c t+x) \sqrt{A_{3}}}{\sqrt{6 B_{1}}}\right)$.
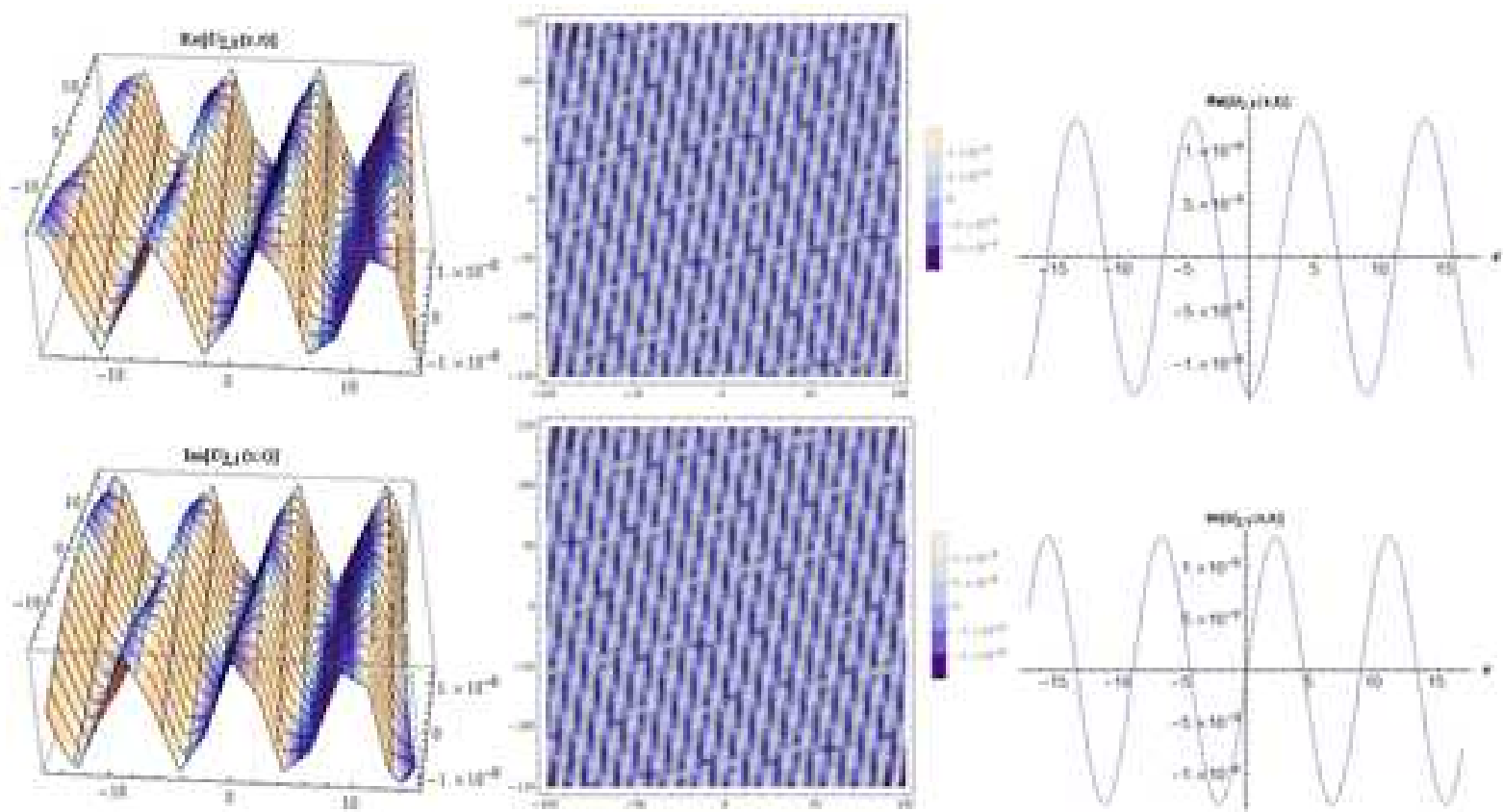

Figure 3: The 3D and contour graphs and $t=1$ for $2 \mathrm{D}$ of Eq. (3.8). 
Family 2:

$$
u_{2,2}(x, t)=\frac{\mu A_{3}\left(-\lambda^{2}+4 \mu\right)}{\left(\lambda \operatorname{Cos}[g(x, t)]-\sqrt{-\lambda^{2}+4 \mu} \operatorname{Sin}[g(x, t)]\right)^{2} B_{1}},
$$

where $g(x, t)=\frac{1}{2} \sqrt{-\lambda^{2}+4 \mu}\left(E+\frac{i(-c t+x) \sqrt{A_{3}}}{\sqrt{6} \sqrt{B_{1}}}\right)$.
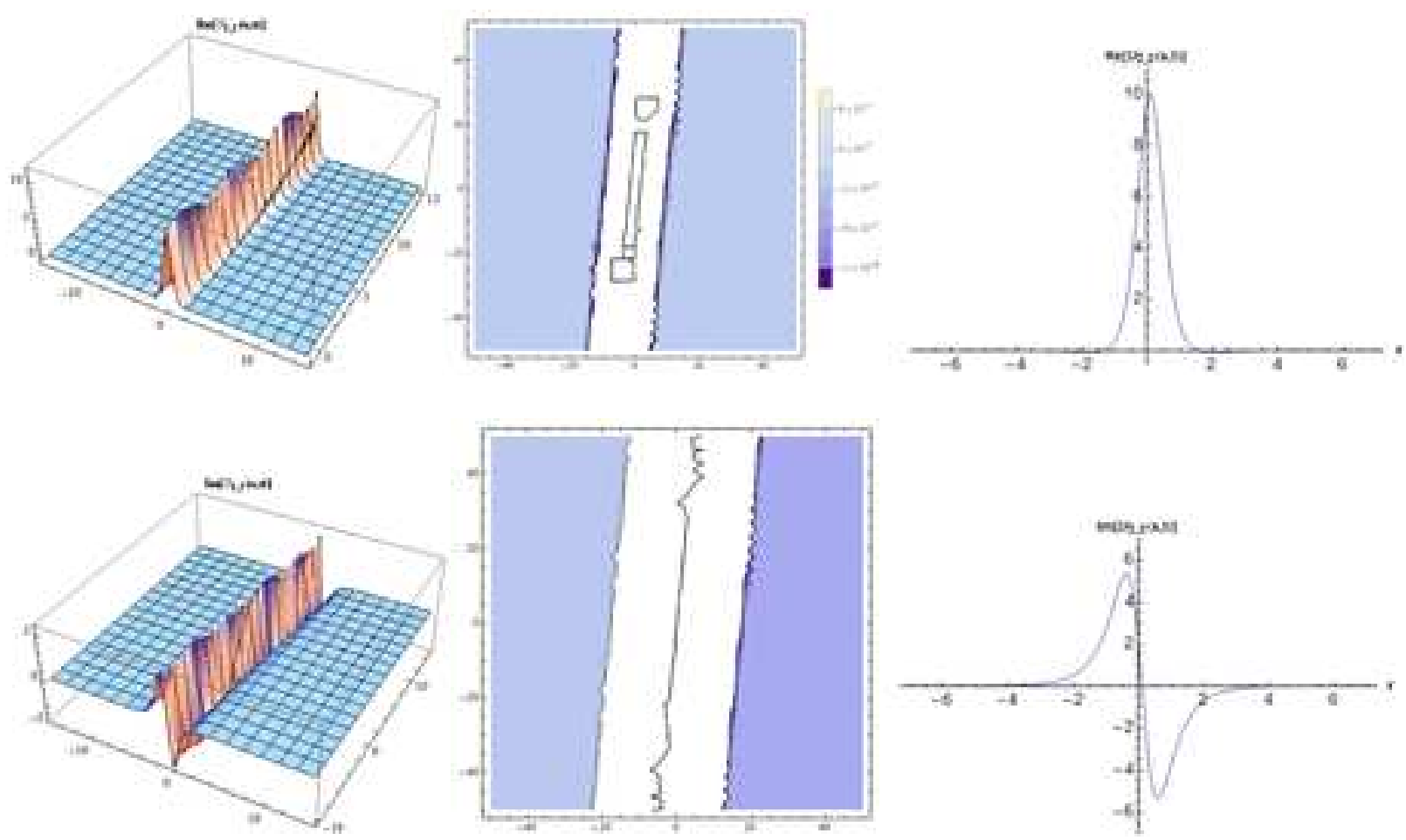

Figure 4: The 3D and contour graphs and $t=1$ for $2 \mathrm{D}$ of Eq. (3.9).

\section{Conclusion}

In this study, we have successfully obtained new travelling wave solutions of the Vakhnenko--Parkes equation by the modified exponential function method. When we compare our results with [36, 42, 44, 49], we saw that all solutions are exactly apart from them. We plotted the 2D-3D and contour surfaces of all travelling wave solutions under the suitable constants. It can be said that the mentioned method is a very effective tool to get analytical solutions of such nonlinear differential equations. Furthermore, to our knowledge, the submitted solutions appear in the literature for the first time. Trigonometric and hyperbolic functions, such as periodic functions, are the functions that best describe the solutions of most problems in physics such as magnetic field, catenary shapes and etc [3-6, 11, 12, 17-20, 22$27,31,32,37,40,41,43,45,46,52,56-60]$. In this regard, we hope that the submitted solutions may be helpful to make out the behavior of frequency waves in physical areas.

\section{References}

[1] T. Akturk, Y. Gurefe, H. Bulut, New function method to the $(\mathrm{n}+1)$-dimensional nonlinear problems, Int. J. Optim. Control. Theor. Appl. IJOCTA, 7 (2017), 234-239. 1 
[2] T. Akturk, Y. Gurefe, H. Bulut, An application of the new function method to the Zhiber-Shabat equation, Int. J. Optim. Control. Theor. Appl. IJOCTA, 7 (2017), 271-274. 1

[3] Z. Avazzadeh, M. H. Heydari, C. Cattani, Legendre wavelets for fractional partial integro-differential viscoelastic equations with weakly singular kernels, The European Physical Journal Plus, 134 (2019), 13 pages. 4

[4] H. M. Baskonus, New acoustic wave behaviors to the Davey-Stewartson equation with power-law nonlinearity arising in fluid dynamics, Nonlinear Dynam., 86 (2016), 177-183.

[5] H. M. Baskonus, New complex and hyperbolic function solutions to the generalized double combined Sinh-Cosh-Gordon equation, AIP Conf. Proc., 1798 (2017), 1-9.

[6] H. M. Baskonus, Complex soliton solutions to the Gilson-Pickering model, Axioms, 8 (2019), 14 pages. 4

[7] H. M. Baskonus, H. Bulut, Analytical studies on the $(1+1)$-dimensional nonlinear dispersive modified Benjamin-BonaMahony equation defined by seismic sea waves, Waves Random Complex Media, 25 (2015), 576-586. 2

[8] H. M. Baskonus, H. Bulut, On the complex structures of Kundu-Eckhaus equation via improved Bernoulli sub-equation function method, Waves Random Complex Media, 25 (2015), 720-728. 1

[9] H. M. Baskonus, H. Bulut, A. Atangana, On the complex and hyperbolic structures of the longitudinal wave equation in a magneto-electro-elastic circular rod, Smart Materials and Structures, 25 (2016), 8 pages. 2

[10] H. M. Baskonus, H. Bulut, T. A. Sulaiman, Investigation of various travelling wave solutions to the extended $(2+1)$ dimensional quantum ZK equation, Eur. Phys. J. Plus , 132 (2017), 8 pages. 1

[11] H. M. Baskonus, H. Bulut, T. A. Sulaiman, New complex hyperbolic structures to the Lonngren-wave equation by using sine-Gordon expansion method, Appl. Math. Nonlinear Sci., 4 (2019), 141-150. 4

[12] H. M. Baskonus, T. A. Sulaiman, H. Bulut, Bright, dark optical and other solitons to the generalized higher-order NLSE in optical Fibers, Optical and Quantum Electronics, 50 (2018), 12 pages. 4

[13] H. Bulut, Application of the modified exponential function method to the Cahn-Allen equation, American Institute of Phys., 1798 (2017), 8 pages. 2

[14] H. Bulut, T. Akturk, Y. Gurefe, Travelling wave solutions of the $(\mathrm{N}+1)$-dimensional sine-cosine-Gordon equation, AIP Conf. Proc., 1637 (2014), 145-149. 1

[15] H. Bulut, T. Akturk, G. Yel, An application of the modified expansion method to nonlinear partial differential equation, Turk. J. Math. Comput. Sci., 10 (2018), 202-206. 2

[16] H. Bulut, G. Yel, H. M. Baskonus, An application of improved Bernoulli sub-equation function method to the nonlinear time-fractional burgers equation, Turk. J. Math. Comput. Sci., 5 (2016), 1-7. 1

[17] C. Cattani, Multiscale analysis of wave propagation in composite materials, Math. Model. Anal., 8 (2003), 267-282. 4

[18] C. Cattani, Harmonic wavelet solutions of the Schrodinger equation, Int. J. Fluid Mech. Res., 30 (2003), $463-472$.

[19] C. Cattani, T. A. Sulaiman, H. M. Baskonus, H. Bulut, Solitons in an inhomogeneous Murnaghan's rod, Eur. Phys. J. Plus, 133 (2018), 11 pages.

[20] C. Cattani, T. A. Sulaiman, H. M. Baskonus, H. Bulut, On the soliton solutions to the Nizhnik-Novikov-Veselov and the Drinfel'd-Sokolov systems, Opt. Quant. Electron., 50 (2018), 11 pages. 4

[21] Y. Chen, Z. Yan, New exact solutions of $(2+1)$-dimensional Gardner equation via the new sine-Gordon equation expansion method, Chaos Solitons Fractals, 26 (2005), 399-406. 1

[22] A. Ciancio, H. M. Baskonus, T. A. Sulaiman, H. Bulut, New structural dynamics of isolated waves via the coupled nonlinear Maccari's system with complex structure, Indian J. Phys., 92 (2018), 1281-1290. 4

[23] F. Dusunceli, Solutions for the Drinfeld-Sokolov equation using an IBSEFM method, MSU J. of Sci., 6 (2018), 505-510.

[24] F. Dusunceli, New exact solutions for the (3+1)-dimensional B-type Kadomtsev-Petviashvili equation, Erzincan University Journal of Science and Technology, 12 (2019), 463-468.

[25] F. Dusunceli, New exponential and complex traveling wave solutions to the Konopelchenko-Dubrovsky model, Adv. Math. Phys., 2019 (2019), 9 pages.

[26] S. M. El-Shaboury, M. K. Ammar, W. M. Yousef, Analytical solutions of the relative orbital motion in unperturbed and in $\mathrm{J}_{2}$-perturbed elliptic orbits, Appl. Math. Nonlinear Sci., 2 (2017), 403-414.

[27] E. I. Eskitaşçioğlu, M. B. Aktas, H. M. Baskonus, New complex and hyperbolic forms for Ablowitz-Kaup-Newell-Segur wave equation with fourth order, Appl. Math. Nonlinear Sci., 4 (2019), 105-112. 4

[28] M. Foroutan, J. Manafian, A. Ranjbaran, Lump solution and its interaction to $(3+1)-D$ potential-YTSF equation, Nonlinear Dyn., 92 (2018), 2077-2092. 1

[29] Y. Gu, W. Yuan, N. Aminakbari, Q. Jiang, Exact solutions of the Vakhnenko-Parkes equation with complex method, J. Funct. Spaces, 2017 (2017), 6 pages. 1

[30] J. H. He, X. H .Wu, Exp-function method for nonlinear wave equations, Chaos Solitons Fractals, 30 (2006), 700-708. 2

[31] M. H. Heydari, M. R. Hooshmandasl, F. M. Maalek Ghaini, C. Cattani, A computational method for solving stochastic Itô-Volterra integral equations based on stochastic operational matrix for generalized hat basis functions, J. Comput. Phys., 270 (2014), 402-415. 4

[32] C. M. Khalique, I. E. Mhlanga, Travelling waves and conservation laws of a $(2+1)$-dimensional coupling system with Korteweg-de Vries equation, Appl. Math. Nonlinear Sci., 3 (2018), 241-253. 4

[33] N. A. Kudryashov, One method for finding exact solutions of nonlinear differential equations, Commun. Nonlinear Sci. Numer. Simul., 17 (2012), 2248-2253. 1

[34] D. Kumar, K. Hosseini, F. Samadani The sine-Gordon expansion method to look for the traveling wave solutions of the Tzitzéica type equations in nonlinear optics , Optik, 149 (2017), 439-446. 1 
[35] C. S. Liu, Trial equation method and its applications to nonlinear evolution equations, Acta Phys. Sinica, 54 (2005), 2505-2509. 1

[36] F. Majid, H. Triki, T. Hayat, O. M. Aldossary, A. Biswas, Solitary wave solutions of the Vakhnenko-Parkes equation, Nonlinear Anal. Model. Control, 17 (2012), 60-66. 1, 4

[37] P. K. Pandey, A new computational algorithm for the solution of second order initial value problems in ordinary differential equations, Appl. Math. Nonlinear Sci., 3 (2018), 167-173. 4

[38] Y. Pandir, Y. Gurefe, U. Kadak, E. Misirli, Classification of exact solutions for some nonlinear partial differential equations with generalized evolution, Abstr. Appl. Anal., 2012 (2012), 16 pages. 1

[39] Y. Pandir, Y. Gurefe, E. Misirli, A new approach to Kudryashov's method for solving some nonlinear physical models, Int. J. Phys. Sci., 7 (2012), 2860-2866. 1

[40] D. Rani, V. Mishra, C. Cattani, Numerical inversion of Laplace transform based on Bernstein operational matrix, Math. Methods Appl. Sci., 41 (2018), 9231-9243. 4

[41] D. Rani, V. Mishra, C. Cattani, Numerical inverse Laplace transform for solving a class of fractional differential equations, Symmetry, 11 (2019), 20 pages. 4

[42] H. Roshid, Md. R. Kabir, R. C. Bhowmik, B. K. Datta, Investigation of Solitary wave solutions for Vakhnenko-Parkes equation via exp-function and $\operatorname{Exp}(\phi(\zeta))$-expansion method, SpringerPlus, 3 (2014), 10 pages. 1, 4

[43] J. J. Rushchitsky, C. Cattani, E. V. Terletskaya, Wavelet analysis of the evolution of a solitary wave in a composite material, Int. Appl. Mech., 40 (2004), 311-318. 4

[44] C. T. Sendi, J. Manafian, H. Mobasseri, M. Mirzazadeh, Q. Zhou, A. Bekir, Application of the ITEM for solving three nonlinear evolution equations arising in fluid mechanics, Nonlinear Dyn., 95 (2019), 669-684. 1, 4

[45] T. A. Sulaiman, H. Bulut, A. Yokus, H. M. Baskonus, On the exact and numerical solutions to the coupled Boussinesq equation arising in ocean engineering, Indian J. Phys., 93 (2019), 647-656. 4

[46] T. A. Sulaiman, A. Yokus, N. Gulluoglu, H. M. Baskonus, Regarding the Numerical and Stability Analysis of the Sharma-Tasso-Olver Equation, ITM Web Conf., 22 (2018), 9 pages. 4

[47] Y. Sun, New travelling wave solutions for Sine-Gordon equation, J. Appl. Math., 2014 (2014), 4 pages. 1

[48] G. Shen, Y. Sun, Y. Xiong, New travelling-wave solutions for Dodd-Bullough equation, J. Appl. Math., 2013 (2013), 5 pages. 1

[49] V. O. Vakhnenko, E. J. Parkes, The two loop soliton solution of the Vakhnenko equation, Nonlinearity, 11 (1998), 14571464. 1,4

[50] V. O. Vakhnenko, E. J. Parkes, Approach in theory of nonlinear evolution equations: the Vakhnenko-Parkes equation, Adv. Math. Phys., 2016 (2016), 39 pages. 1

[51] A. M. Wazwaz, Multiple-soliton solutions for the KP equation by Hirota's bilinear method and by the tanh-coth method, Appl. Math. Comput., 190 (2007), 633-640. 1

[52] E. W. Weisstein, CRC concise encyclopedia of mathematics, CRC Press, (2002). 4

[53] F. Xu, Application of Exp-function method to symmetric regularized long wave (SRLW) equation, Phys. Lett., 372 (2008), 252-257. 2

[54] X. F. Yang, Z. C. Deng, Y. Wei, A Riccati-Bernoulli sub-ODE method for nonlinear partial differential equations and its application, Adv. Difference Equ., 2015 (2015), 17 pages. 1

[55] G. Yel, H. M. Baskonus, H. Bulut, Novel archetypes of new coupled Konno-Oono equation by using sine-Gordon expansion method, Opt. Quant. Electron., 49 (2017), 10 pages. 1

[56] G. Yel, H. M. Baskonus, H. Bulut, Regarding on the some novel exponential travelling wave solutions to the Wu-Zhang system arising in nonlinear water wave model, Indian J. Phys., 93 (2019), 1031-1039. 4

[57] A. Yokus, Comparison of Caputo and conformable derivatives for time-fractional Korteweg-de Vries equation via the finite difference method, Internat. J. Modern Phys., 32 (2018), 12 pages.

[58] A. Yokus, S. Gulbahar, Numerical solutions with linearization techniques of the fractional Harry Dym equation, Appl. Math. Nonlinear Sci., 4 (2019), 35-41.

[59] A. Yokus, T. A. Sulaiman, M. T. Gulluoglu, H. Bulut, Stability analysis, numerical and exact solutions of the $(1+1)-$ dimensional NDMBBM equation, ITM Web Conf., 22 (2018), 10 pages.

[60] A. Yokus, M. Tuz, An application of a new version of $\left(\mathrm{G}^{\prime} / \mathrm{G}\right)$-expansion method, In AIP Conf. Proc., 1798 (2017), 7 pages. 4

[61] Y. Yujian, S. Junquan, S. Shoufeng, D. Yanmei, New coherent structures of the Vakhnenko-Parkes equation, Results in Physics, 2 (2012), 170-174. 1 